\title{
Cell Respiration—Energy Production of Cells
}

\author{
Jianing $\mathrm{Hu}$
}

\author{
KangChiao International School, Shanghai, China, 200135 \\ *Corresponding author. Email: jared705823@outlook.com
}

\begin{abstract}
Cellular respiration has always been an important topic in the history of biology. In the past, many scientists have studied this topic. Decades ago, humans first found mitochondria in the cell, then start the big research of cellular respiration. When the research becomes deeper, they divide the big pathway into smaller processes, such as glycolysis, pyruvate oxidation, citric acid cycle, and oxidative phosphorylation. One method of researching cellular respiration is to cultivate cells in a petri-dish and give them different kinds of nutrition, such as glucose, protein, or lipids. Then observe the growth of the cells. The research is very meaningful to humans because this can make scientists know more about the human body. It can explain why humans breathe oxygen in and exhale carbon dioxide. The research result can also be widely used in pharmaceuticals. It can also help experts to make appropriate diets for humans. The research in this field has never been stopped. Up to now, the research in cellular respiration has been very mature. Biologists have found the input, process, and output of cellular respiration.
\end{abstract}

Keywords: Glucose, Adenosine Triphosphate (ATP), Mitochondria, Nicotinamide Adenine Dinucleotide. (NADH), Enzyme.

\section{THE INTRODUCTION TO CELLULAR RESPIRATION}

Cellular respiration has always been an important topic in the history of biology. Many scholars studied this topic in the past. Decades ago, man first found mitochondria in a cell and then began a great deal of research into cellular respiration. As research gets deeper, it divides the long path into smaller processes such as glycolysis, pyruvate oxidation, citric acid cycle, and oxidative phosphorylation. One method for studying cellular respiration is to culture cells in a Petri dish and feed them various types of food, such as glucose, protein, or lipids. Then watch the growth of the cells. Research is important to humans because it can help scientists learn more about the human body. This may explain why a person inhales oxygen and exhales carbon dioxide. The research result can also find wide application in pharmaceuticals. It can also help professionals design a suitable diet for a person. Research in this area has never stopped. The research in this field has never been stopped. The research in cellular respiration is very mature. Biologists have found the process of cellular respiration and its output. Cellular respiration can occur in all types of human cells, therefore, the author considers it is the most reaction in the human body. In history, many scientists have studied this topic. In this essay, the author did a lot of research and put everything together to introduce a complete pathway of cellular respiration, In this essay, other three processes in cellular respiration, pyruvate oxidation, Krebs cycle, oxidative phosphorylation, and the overview of the whole process of cellular respiration is introduced.

\section{THE BRIEF INFORMATION OF CELLULAR RESPIRATION}

\subsection{The definition of cellular respiration}

Cellular respiration, which means the respiration of cells, is an essential activity of metabolism within a cell. During cellular respiration, oxygen and organic molecules are consumed, carbon dioxide and ATP are produced. During the process, Oxygen is converted into carbon dioxide. To promote this process, human use the lung to respirate, which is a gas-exchange process, provide oxygen and excrete carbon dioxide for the cells. As long as a person is breathing, he is doing cellular respiration. 


\subsection{The origin and development of cellular respiration}

The structure of a eukaryote cell is very complicated, it contains many organelles. The nucleus contains the genetic information of the cell in the form of DNA or chromosomes and thus, controls cell growth and multiplication[1]. Lysosome has a high concentration of hydrogen proton in it, its function to decompose wastes in the cell. The ribosome can synthesize polypeptides according to the mRNA sequence. The endoplasmic reticulum process the polypeptide into protein and then send it to the Golgi apparatus and finally send it out of the cell. All these organelles need the energy to work, if there is no energy, the cell will die quickly. Sufficient energy supply in our cell should give credit to mitochondria. Mitochondria is the power station of a cell, it can continuously produce ATP. ATP is the main energy source in our cells. Its full name is adenosine triphosphate. This means it consists of one adenine, one ribose, and three phosphate group. It can release energy by giving away one phosphate group, becoming ADP, full name is adenosine diphosphate. Mitochondria have an inner and outer membrane, with an intermembrane space between them. The outer membrane contains proteins known as porins, which allow the movement of ions into and out of the mitochondrion [2]. The symbiosis hypothesis suggests that mitochondria were once a prokaryote, accidentally captured by our cells and stayed in. This hypothesis is credible because it is supported by many pieces of evidence, for example, it has its genetic materials and ribosomes. One interesting fact is mitochondrial DNA can also affect phenotype, some disease is mitochondrial inheritance. But mitochondrial DNA can only be inherited by the mother.

\section{THE INTRODUCTION TO THE ENERGY PRODUCTION OF CELLS}

As the most important organelle in cell respiration, the discovery of mitochondria symbolizes the start of discovering cell respiration. Mitochondria were first discovered in 1857 by a Swiss anatomist and physiologist, Albert von Kolliker. Kölliker became a professor of physiology and comparative anatomy at the University of Zürich in 1844 [3]. He found the granulelike structure in muscle cells. Then, Richard Altmann did further research on mitochondria and call it "bioblast". He hypothesized mitochondria are the basic unit of cell activity. In 1898, Carl Benda created the term "mitochondria". Mitochondria mean thread-like granule, "mito" means thread, "chondros" means granule. In 1937, Sir Hen Adolf Krebs discovered the Citric acid cycle or the Krebs cycle. In 1953, he got the Nobel prize for medicine because of this discovery. The discovery of glycolysis started in the 1850 s, a French wine industry want to find out why wine sometimes doesn't turn into alcohol. Louis Pasteur finds out that yeast consumes less glucose under aerobic conditions, compared to anaerobic conditions. In the 1940s, Otto Meyerhof and many other biochemists finally completed the pathway of glycolysis. Otto Meyerhof, German biochemist, and co-recipient, with Archibald V. Hill, of the 1922 Nobel Prize for Physiology or Medicine for research on the chemical reactions of metabolism in muscle[4]. Humans have trillions of cells in our bodies. For example erythrocytes, its function is to carry oxygen molecule and deliver it to all the places in the body. But why does our body need such a large amount of oxygen? This question can be answered by explaining the process of cell energy production.

\subsection{The process of energy production of cells}

This is the first step in energy production, which happens in the cytosol. Glycolysis break one glucose molecule into two pyruvate molecules. It required 2 ATP to start the process and it would finally produce 2 NADH and 4 ATP, a net gain of 2 ATP and 2 NADH. $\mathrm{NADH}$ is another energy-carrying molecule in our body, it has almost the same function as ATP in glycolysis and citric acid cycle. It will provide hydrogen protons for oxidative phosphorylation. Its full name is nicotinamide adenine dinucleotide, which consists of two nucleotides. It can release energy by giving away one hydrogen proton, becoming NAD + . Pyruvate is a 3 carbon molecule, it cannot enter the citric acid cycle directly. Pyruvate oxidation is a process that converts pyruvate into Acetyl-CoA. Acetyl is a two-carbon molecule. CoA is a coenzyme, its full name is Coenzyme A, which can help acetyl to enter the citric acid cycle. When pyruvate enters the mitochondria, this process happens. It will release one carbon dioxide, change one NAD+ into NADH. After pyruvate oxidation, Acetyl-CoA will enter the mitochondrial matrix. First, Acetyl-CoA will bind with oxaloacetate, a four-carbon molecule, and form a six-carbon citric molecule. Then, coenzyme A will leave and the citric molecule will enter the citric acid cycle. The whole process will produce $3 \mathrm{NADH}, 1$ FADH2, 1 ATP, 2 $\mathrm{CO} 2$. The citric molecule will lose 2 carbons and become oxaloacetate, ready to bind with another Acetyl-CoA and start the critic acid cycle. CO2 will directly cross the phospholipid bilayer since it is nonpolar and small in size. NADH and FADH2 cannot directly be used by our cell, they will release the electron and form a hydrogen proton gradient in the next step. Oxidative phosphorylation is the most complicated process in cell respiration, but also the most important because it produces the most amount of ATP. First, NADH will attach to protein complex 1, donate an electron, become NAD + and $\mathrm{H}+$. Then, FADH2 will donate two electrons to protein complex 2, then these electrons will meet the previous electron and 
travel to protein complex 3 and 4 together. At last, the three electrons will induce 2 hydrogen protons to bind with half of an oxygen molecule to form a water molecule. In this process, protein complexes 1,3 , and 4 will be promoted by the kinetic energy of the electrons, and each transports one hydrogen proton from the mitochondrial matrix to intermembrane space, gradually forming an electrical gradient. After the gradient is formed, hydrogen protons start to pass the inner membrane automatically. There are two kinds of membrane transport in biology, active transport, and passive transport. Active transport move particles against the gradient, therefore, requires energy to progress, such as endocytosis, exocytosis. In contrast, passive transport doesn't require energy, particles move automatically from higher gradient to lower gradient, like a dam releasing water. Examples of passive transport are osmosis, diffusion, and facilitated diffusion. In oxidative phosphorylation, hydrogen protons automatically pass through the ATP synthase. The kinetic energy can cause the enzyme to rotate, combine an ADP with a phosphate group, form an ATP. Cellular respiration is dependent on the existence of an electrochemical proton gradient across the inner mitochondrial membrane [5].

\subsection{The implication of energy production of cells}

With the research of cellular respiration becoming deeper, the result becomes more and more useful. The research of cellular respiration helps the research of photosynthesis, which is approximately the inverse process of cellular respiration. Biologists found two photoreceptors in the chlorophyll of plant cells. Consequently, found that light of different wavelengths can affect the growth of a specific plant. Now, this method is widely used in agriculture in that peasants use the light of different colors to illuminate the vegetable shed, causing the crops to grow faster and bigger. Result in the research of cellular respiration are also useful in solving environmental issues. During the past decades, humans notice that the average temperature of the earth is increasing over years. Originally, they think this was the human industry's fault. However, several years ago, they found that the raise of livestock emit a lot of greenhouse gases to the atmosphere. The livestock needs to do cellular respiration and exhale a lot of carbon dioxides. Humans used to produce electricity by using wind, water power, or biomass. These traditional ways are all harmful to the environment. Recently, scientists found a way, they use water to produce electricity. Unlike the traditional way which builds a dam to damage the ecosystem. Hydrogen fuel cells produce electricity by combining hydrogen and oxygen atoms[6]. The hydrogen reacts with oxygen across an electrochemical cell similar to that of a battery to produce electricity, water, and small amounts of heat[6]. The input and output are both pure water. This is a bionic technique, credit to the research of cellular respiration.

\section{THE ADVANTAGE AND DISADVANTAGES OF ENERGY PRODUCTION OF CELLS}

In this essay, cellular respiration which consumes glucose is introduced. In the human body, glucose is the first source of energy to be used. However, when a person is starving, he cannot eat enough food for a long period, the body system can still operate with sufficient energy. In this condition, cells will change their source of energy from glucose to lipids, which are stored in adipose tissue in another part of the body, delivered in blood vessels to the whole body. The mechanism can break down glycerol molecules using another pathway until it binds with coenzyme A and become acetyl-CoA, similar to glucose. Then, it can promote the citric acid cycle and oxidative phosphorylation. When lipids were consumed, protein can also be a source of energy. Similar to glycerol, the amino acid can become acetyl$\mathrm{CoA}$ as well. When all proteins and lipids are consumed, the person will be extremely thin, he lost all the fats and muscles. Under this condition, the cell can consume nucleic acid as a source of energy. These macromolecules can all be broken down into acetyl$\mathrm{CoA}$ is because they are all carbon-based organic molecules. This advantage of cellular respiration can save a human's life under extreme conditions.

Cellular respiration cause risks of disease to human beings. For example, Alzheimer's disease is linked to those cells which can use glucose without going into oxidative phosphorylation. Another disadvantage of cellular respiration is that tumor cells can also have this energy production process, therefore, they can grow and duplicate very fast, much faster than normal cells. When mutation cells grow fast, they can quickly become a tumor and use the energy of a certain organ it grows on. The cellular respiration that happens in tumor cells could kill a human if that human got cancer.

\section{CONCLUSION}

Cellular respiration is an important process in the human body. It can provide energy for the cell to operate. During cellular respiration, macromolecules are decomposed into Acetyl-CoA and create a hydrogen proton gradient. In this essay, cellular respiration by glucose is introduced. During cell respiration, 38 ATPs can be produced by one glucose, 2 from glycolysis, 2 from the Krebs cycle, and 34 from oxidative phosphorylation. For other macromolecule such as protein, lipid, and nucleic acid, the process of the citric acid cycle and oxidative phosphorylation is the same as glucose. The essay did not discuss the use of energy, 
and the ATP molecules produced by the cell are widely used in the human body. This is also a topic which scientists are busy researching today. There are a lot of protein channels on the cell membrane, and these protein channels are responsible for facilitated diffusion. The working mechanism of these protein channels is using the energy from ATP to promote the open or close. These protein channels are "the gate of a cell". The author predicts that this will be the future of researching the use of ATP.

\section{REFERENCES}

[1] Shaziya Allarakha, M. D. (2021, February 12). What are the 3 functions of the nucleus? MedicineNet. Retrieved November 3, 2021, from https://www.medicinenet.com/what_are_the_3_fun ctions_of_the_nucleus/article.htm.

[2] Badrick, A. (2020, May 9). Mitochondria - structure - function. TeachMePhysiology. Retrieved November 3, 2021, from https://teachmephysiology.com/histology/cellstructures/mitochondria/.

[3] Encyclopædia Britannica, inc. (n.d.). Rudolf Albert von Kölliker. Encyclopædia Britannica. Retrieved November 3, 2021, from https://www.britannica.com/biography/RudolfAlbert-von-Kolliker.

[4] :Encyclopædia Britannica, inc. (n.d.). Otto Meyerhof. Encyclopædia Britannica. Retrieved November 3, 2021, from https:/www.britannica.com/biography/OttoMeyerhof.

[5] MEFANET, C. and S. medical faculties network. (n.d.). The electrochemical proton gradient. WikiLectures. Retrieved November 3, 2021, from https://www.wikilectures.eu/w/The_Electrochemic al_Proton_Gradient.

[6] U.S. Energy Information Administration - EIA independent statistics and analysis. Use of hydrogen - U.S. Energy Information Administration (EIA). (n.d.). Retrieved November 8, 2021, from https://www.eia.gov/energyexplained/hydrogen/us e-of-hydrogen.php. 